\title{
Impact of higher-order coherences on the carrier dynamics in Landau-quantized graphene
}

\author{
Florian Wendler* and Ermin Malic \\ Department of Applied Physics, Chalmers University of Technology, Göteborg, Sweden;
}

\begin{abstract}
We investigate the carrier dynamics in Landau-quantized graphene within the density matrix formalism. In particular, we focus on the carrier-light interaction addressing the impact of higher-order polarizations beyond the optical selection rules. We find that these terms are in general negligible, however, there are regimes, where they even become crucial for the carrier dynamics. Our calculations show that for short excitation pulses, very small Landau level broadenings, and certain configurations of magnetic field strength, Fermi energy, and excitation energy, higher-order polarizations need to be taken into account.
\end{abstract}

Keywords: Landau-quantized graphene, carrier dynamics, carrier-light interaction, higher-order polarizations, density matrix theory

\section{INTRODUCTION}

Since the groundbreaking experiments on the exfoliation of graphene within the scotch-tape method in $2004,{ }^{1}$ graphene has attracted much attention, ${ }^{2}$ and has been discussed as a new material for a variety of technological applications. ${ }^{3-10}$ A profound understanding of the carrier dynamics in photo-excited graphene is the key to exploit the full potential of this interesting material in the field of optoelectronics. ${ }^{11}$ While the ultrafast relaxation dynamics in graphene has been extensively studied, ${ }^{12-27,27-36}$ the research investigating the dynamics of charge carriers in graphene subject to an external magnetic field has recently started. ${ }^{37-43}$ In an external magnetic field, the energy is quantized into Landau levels (LLs). ${ }^{44}$ Here, unlike in an usual semiconductor, the energetic spacing between the discrete energy levels is non-equidistant resulting in an interesting behavior of photo-excited charge carriers. ${ }^{38,40,41}$

In this Article, we investigate the carrier-light interaction in Landau-quantized graphene based on the density matrix formalism resulting in optical Bloch equations. ${ }^{45-48}$ The latter present a coupled system of differential equations describing the density matrix $\sigma_{i \rightarrow f}(t)=\left\langle a_{f}^{\dagger} a_{i}\right\rangle(t)$, where $a_{i}^{\dagger}$ and $a_{i}$ are fermionic creation and annihilation operators of the state $i$. While the diagonal elements of the density matrix $\sigma_{i \rightarrow f=i}=\rho_{i}$ describe carrier occupation probabilities and are important e.g. to model pump probe experiments ${ }^{49}$ the off-diagonal elements $\sigma_{i \rightarrow f \neq i}=p_{i \rightarrow f}$, also called coherences or microscopic polarizations, describe the optical excitation of the material. Considering low-energetic Landau levels with the indices $n=0,1,2, \ldots, N$ in the conduction and valence bands $(\lambda=v, c)$, the density matrix has $(2 N+1)^{2}$ entries including the zeroth LL $n=0$ that belongs to both bands. The computational effort for the numeric solution of the Bloch equations can be considerably reduced by taking into account only off-diagonal elements $\sigma_{n \rightarrow n \pm 1}(t)$ that fulfill the optical selection rules. In this work, we show that in certain situations this approximation is not valid requiring higher-order off-diagonal elements $\sigma_{n \rightarrow n \pm 2}(t)$ to be included.

\section{OPTICAL BLOCH EQUATIONS}

Here, we briefly present the derivation of optical Bloch equations for Landau-quantized graphene. The starting point is the Hamilton operator

$$
H=\sum_{i} \epsilon_{i} a_{i}^{\dagger} a_{i}+i \hbar \sum_{i, f} \Omega_{i \rightarrow f} a_{f}^{\dagger} a_{i}
$$

*florian.wendler@tu-berlin.de

Terahertz Emitters, Receivers, and Applications VI, edited by

Manijeh Razeghi, Alexei N. Baranov, John M. Zavada, Dimitris Pavlidis, Proc. of SPIE

Vol. 9585, 95850D - (C) 2015 SPIE · CCC code: 0277-786X/15/\$18 - doi: 10.1117/12.2186880

Proc. of SPIE Vol. 9585 95850D-1 


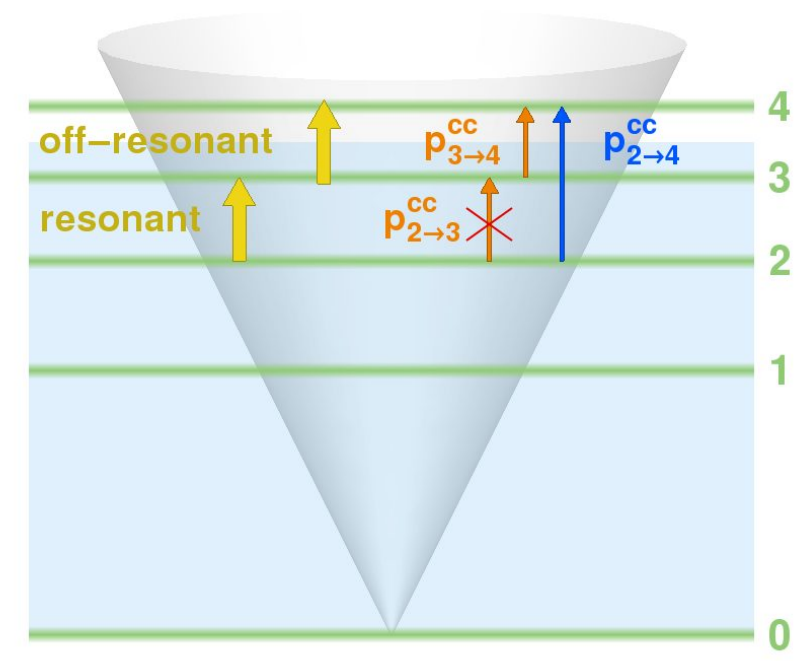

Figure 1. Sketch illustrating a situation in which higher-order polarizations are important. The low-energetic Landau levels in the conduction band of graphene are shown (green lines) with the Dirac cone in the background. The Fermi energy is assumed to lie between $\mathrm{LL}_{3}$ and $\mathrm{LL}_{4}$, hence, the occupation of all LLs up to $\mathrm{LL}_{3}$ is approximately $100 \%$ (blueshaded area). Resonantly pumping the transition $2 \rightarrow 3$, no electrons can be optically excited from $\mathrm{LL}_{2}$ to $\mathrm{LL}_{3}$, since $\mathrm{LL}_{3}$ is already completely occupied. Instead, the off-resonant transition $3 \rightarrow 4$ is induced. Moreover, since the lowest order polarization $p_{2 \rightarrow 3}$ is strongly suppressed, higher-order coherences $p_{2 \rightarrow 4}$ become important.

where the first term is the free energy depending on the Landau level dispersion $\epsilon_{n}^{\lambda}=\lambda v_{\mathrm{F}} \sqrt{2 \hbar n e_{0} B}$ with the elementary charge $e_{0}$ and the magnetic field $B$. The second term describes the carrier-light interaction expressed by the Rabi frequency $\Omega_{i \rightarrow f}(t)=\frac{e_{0}}{m_{0}} \mathbf{M}_{i \rightarrow f} \cdot \mathbf{A}(t)$ that is the product of the optical matrix element $\mathbf{M}_{i \rightarrow f}$ and the vector potential $\mathbf{A}(t)$. An analytic expression for the optical matrix element can be derived by exploiting tight-binding wave functions in combination with the Peierls substition considering the impact of the magnetic field ${ }^{40,50}$

$$
\mathbf{M}_{i \rightarrow f}=i \delta_{\xi_{i}, \xi_{f}} \delta_{m_{i}, m_{f}} \frac{\alpha_{n_{i}} \alpha_{n_{f}} m_{0} v_{\mathrm{F}}}{2 \sqrt{2} \hbar}\left[\lambda_{i} \hat{\boldsymbol{\epsilon}}^{-} \delta_{n_{f}, n_{i}-1}+\lambda_{f} \hat{\boldsymbol{\epsilon}}^{+} \delta_{n_{f}, n_{i}+1}\right]=-\mathbf{M}_{f \rightarrow i}^{*} .
$$

Here, $\xi= \pm 1$ is the valley index ${ }^{44} m=0,1, \ldots$ is a quantum number expressing the Landau level degeneracy, ${ }^{44}$ the constant $\alpha_{n=0}$ equals $\sqrt{2}$ for $n=0$ and is 1 otherwise, $m_{0}$ denotes the free electron mass, $v_{\mathrm{F}} \approx 1 \mathrm{~nm} / \mathrm{fs}$ is the Fermi velocity, ${ }^{51}$ and $\hat{\boldsymbol{\epsilon}}^{ \pm}=\left(\hat{\mathbf{e}}_{x} \mp i \hat{\mathbf{e}}_{y}\right) / \sqrt{2}$ are Jones vectors describing left $(+)$ and right $(-)$ circularly polarized light. ${ }^{52}$ The optical selection rules are expressed by the Kronecka deltas $\delta_{n_{f}, n_{i} \pm 1}$. As a consequence, only polarizations of the form $p_{n \rightarrow n \pm 1}$ are directly driven by an optical excitation and in general it is a good approximation to neglect higher-order coherences. The next order of such coherences is either $p_{n \rightarrow n \pm 2}$ or $p_{n \rightarrow n}^{\mathrm{vc}}$. While the latter always corresponds to a transition between the valence (v) and the conduction (c) band, the first can be both intra- and interband Landau level transitions. These higher-order terms are negligible, as long as the build-up of the directly optically driven polarization $p_{n \rightarrow n \pm 1}$ is not strongly suppressed due to Pauli blocking, cf. Fig. 1.

To investigate the carrier-light coupling and the influence of higher-order polarizations, we derive the optical Bloch equations for Landau-quantized graphene. Exploiting the Heisenberg equation of motion, we obtain the most general form of these equations ${ }^{11,53}$

$$
\dot{\sigma}_{i \rightarrow f}(t)=\left[i \triangle \omega_{i f}-\gamma\right] \sigma_{i \rightarrow f}(t)+\sum_{l}\left[\Omega_{l \rightarrow i}(t) \sigma_{l \rightarrow f}(t)-\Omega_{f \rightarrow l}(t) \sigma_{i \rightarrow l}(t)\right] .
$$

Here, $\hbar \triangle \omega_{i f}=\left(\epsilon_{f}-\epsilon_{i}\right)$ is the transition energy and $\gamma$ corresponds to a dephasing describing the impurityinduced Landau level broadening $\Gamma=\hbar \gamma$. The optical interaction conserves all quantum numbers characterizing the state $l$ except for the band index $\lambda_{l}$ and the Landau level index $n_{l}$, cf. Eq. 2. Taking into account the optical 
selection rules that are reflected by the Kronecker deltas $\delta_{n_{f}, n_{i} \pm 1}$ in Eq. 3, the sum over the Landau level index is reduced to a few terms. Fixing $i=f=n$ and $\lambda$ either to $c$ or $v$, we perform the appearing sum over $\lambda_{l}$ and obtain the first Bloch equations describing the carrier occupation probabilities $\rho_{n}^{\lambda}$

$$
\begin{aligned}
& \dot{\rho}_{n}^{c}=-2 \operatorname{Re}\left[-\left(\Omega_{n \rightarrow n+1}^{c c} p_{n \rightarrow n+1}^{c c}\right)^{*}+\Omega_{n-1 \rightarrow n}^{c c} p_{n-1 \rightarrow n}^{c c}+\Omega_{n+1 \rightarrow n}^{v c} p_{n+1 \rightarrow n}^{v c}+\Omega_{n-1 \rightarrow n}^{v c} p_{n-1 \rightarrow n}^{v c}\right], \\
& \dot{\rho}_{n}^{v}=-2 \operatorname{Re}\left[\Omega_{n+1 \rightarrow n}^{v v} p_{n+1 \rightarrow n}^{v v}-\left(\Omega_{n \rightarrow n-1}^{v v} p_{n \rightarrow n-1}^{v v}\right)^{*}-\left(\Omega_{n \rightarrow n+1}^{v c} p_{n \rightarrow n+1}^{v c}\right)^{*}-\left(\Omega_{n \rightarrow n-1}^{v c} p_{n \rightarrow n-1}^{v c}\right)^{*}\right] .
\end{aligned}
$$

Fixing $i=n, f=n \pm 1$, we obtain for the optically driven polarizations $p_{n \rightarrow n \pm 1}^{\lambda \lambda^{\prime}}$ :

$$
\begin{aligned}
\dot{p}_{n \rightarrow n+1}^{c c} & =\left(i \triangle \omega_{n \rightarrow n+1}^{c c}-\gamma\right) p_{n \rightarrow n+1}^{c c}+\left[\left(\Omega_{n \rightarrow n+1}^{c c}\right)^{*}\left[\rho_{n}^{c}-\rho_{n+1}^{c}\right]+\left(\Omega_{n \rightarrow n+1}^{v c} p_{n \rightarrow n}^{v c}\right)^{*}+\Omega_{n+1 \rightarrow n}^{v c} p_{n+1 \rightarrow n+1}^{v c}\right. \\
& \left.+\Omega_{n-1 \rightarrow n}^{c c} p_{n-1 \rightarrow n+1}^{c c}-\Omega_{n+1 \rightarrow n+2}^{c c} p_{n \rightarrow n+2}^{c c}+\Omega_{n-1 \rightarrow n}^{v c} p_{n-1 \rightarrow n+1}^{v c}+\left(\Omega_{n+2 \rightarrow n+1}^{v c} p_{n+2 \rightarrow n}^{v c}\right)^{*}\right] \\
\dot{p}_{n \rightarrow n-1}^{v v} & =\left(i \triangle \omega_{n \rightarrow n-1}^{v v}-\gamma\right) p_{n \rightarrow n-1}^{v v}+\left[\left(\Omega_{n \rightarrow n-1}^{v v}\right)^{*}\left[\rho_{n}^{v}-\rho_{n-1}^{v}\right]-\Omega_{n-1 \rightarrow n}^{v c} p_{n \rightarrow n}^{v c}-\left(\Omega_{n \rightarrow n-1}^{v c} p_{n-1 \rightarrow n-1}^{v c}\right)^{*}\right. \\
& \left.+\Omega_{n+1 \rightarrow n}^{v v} p_{n+1 \rightarrow n-1}^{v v}-\Omega_{n-1 \rightarrow n-2}^{v v} p_{n \rightarrow n-2}^{v v}-\left(\Omega_{n \rightarrow n+1}^{v c} p_{n-1 \rightarrow n+1}^{v c}\right)^{*}-\Omega_{n-1 \rightarrow n-2}^{v c} p_{n \rightarrow n-2}^{v c}\right] \\
\dot{p}_{n \rightarrow n+1}^{v c} & =\left(i \triangle \omega_{n \rightarrow n+1}^{v c}-\gamma\right) p_{n \rightarrow n+1}^{v c}+\left[\left(\Omega_{n \rightarrow n+1}^{v c}\right)^{*}\left[\rho_{n}^{v}-\rho_{n+1}^{c}\right]+\left(\Omega_{n \rightarrow n+1}^{c c}\right)^{*} p_{n \rightarrow n}^{v c}+\Omega_{n+1 \rightarrow n}^{v v} p_{n+1 \rightarrow n+1}^{v c}\right. \\
& -\left(\Omega_{n \rightarrow n-1}^{v c}\right)^{*} p_{n-1 \rightarrow n+1}^{c c}+\left(\Omega_{n+2 \rightarrow n+1}^{v c} p_{n+2 \rightarrow n}^{v v}-\left(\Omega_{n \rightarrow n-1}^{v v}\right)^{*} p_{n-1 \rightarrow n+1}^{v c}-\Omega_{n+1 \rightarrow n+2}^{c c} p_{n \rightarrow n+2}^{v c}\right] \\
\dot{p}_{n \rightarrow n-1}^{v c} & =\left(i \triangle \omega_{n \rightarrow n-1}^{v c}-\gamma\right) p_{n \rightarrow n-1}^{v c}+\left[\left(\Omega_{n \rightarrow n-1}^{v c}\right)^{*}\left[\rho_{n}^{v}-\rho_{n-1}^{c}\right]-\Omega_{n-1 \rightarrow n}^{c c} p_{n \rightarrow n}^{v c}-\left(\Omega_{n \rightarrow n-1}^{v v}\right)^{*} p_{n-1 \rightarrow n-1}^{v c}\right. \\
& \left.-\left(\Omega_{n \rightarrow n+1}^{v c} p_{n-1 \rightarrow n+1}^{c c}\right)^{*}+\left(\Omega_{n-2 \rightarrow n-1}^{v c}\right)^{*} p_{n \rightarrow n-2}^{v v}+\Omega_{n+1 \rightarrow n}^{v v} p_{n+1 \rightarrow n-1}^{v c}+\left(\Omega_{n-2 \rightarrow n-1}^{c c}\right)^{*} p_{n \rightarrow n-2}^{v c}\right] .
\end{aligned}
$$

Examining Eqs. (6)-(9), we observe that the optically driven polarizations also couple to higher-order polarizations of the forms $p_{n \rightarrow n}^{v c}$ as well as $p_{n \rightarrow n \pm 2}$. To investigate the importance of these terms, we derive the corresponding equations of motion by accordingly performing the sum in Eq. 3

$$
\begin{aligned}
\dot{p}_{n \rightarrow n}^{v c}= & \left(i \triangle \omega_{n \rightarrow n}^{v c}-\gamma\right) p_{n \rightarrow n}^{v c} \\
+ & {\left[-\left(\Omega_{n \rightarrow n+1}^{v c} p_{n \rightarrow n+1}^{c c}\right)^{*}-\left(\Omega_{n \rightarrow n-1}^{v c}\right)^{*} p_{n-1 \rightarrow n}^{c c}+\left(\Omega_{n+1 \rightarrow n}^{v c} p_{n+1 \rightarrow n}^{v v}\right)^{*}+\left(\Omega_{n-1 \rightarrow n}^{v c}\right)^{*} p_{n \rightarrow n-1}^{v v}\right.} \\
- & \left.\Omega_{n \rightarrow n+1}^{c c} p_{n \rightarrow n+1}^{v c}+\Omega_{n+1 \rightarrow n}^{v v} p_{n+1 \rightarrow n}^{v c}+\left(\Omega_{n-1 \rightarrow n}^{c c}\right)^{*} p_{n \rightarrow n-1}^{v c}-\left(\Omega_{n \rightarrow n-1}^{v v}\right)^{*} p_{n-1 \rightarrow n}^{v c}\right], \\
\dot{p}_{n \rightarrow n+2}^{c c}= & \left(i \triangle \omega_{n \rightarrow n+2}^{c c}-\gamma\right) p_{n \rightarrow n+2}^{c c} \\
+ & {\left[\left(\Omega_{n+1 \rightarrow n+2}^{c c}\right)^{*} p_{n \rightarrow n+1}^{c c}-\left(\Omega_{n \rightarrow n+1}^{c c}\right)^{*} p_{n+1 \rightarrow n+2}^{c c}+\left(\Omega_{n+1 \rightarrow n+2}^{v c} p_{n+1 \rightarrow n}^{v c}\right)^{*}+\Omega_{n+1 \rightarrow n}^{v c} p_{n+1 \rightarrow n+2}^{v c}\right], } \\
\dot{p}_{n \rightarrow n-2}^{v v}= & \left(i \triangle \omega_{n \rightarrow n-2}^{v v}-\gamma\right) p_{n \rightarrow n-2}^{v v} \\
+ & {\left[\left(\Omega_{n-1 \rightarrow n-2}^{v v}\right)^{*} p_{n \rightarrow n-1}^{v v}-\left(\Omega_{n \rightarrow n-1}^{v v}\right)^{*} p_{n-1 \rightarrow n-2}^{v v}-\Omega_{n-2 \rightarrow n-1}^{v c} p_{n \rightarrow n-1}^{v c}-\left(\Omega_{n \rightarrow n-1}^{v c} p_{n-2 \rightarrow n-1}^{v c}\right)^{*}\right], }
\end{aligned}
$$



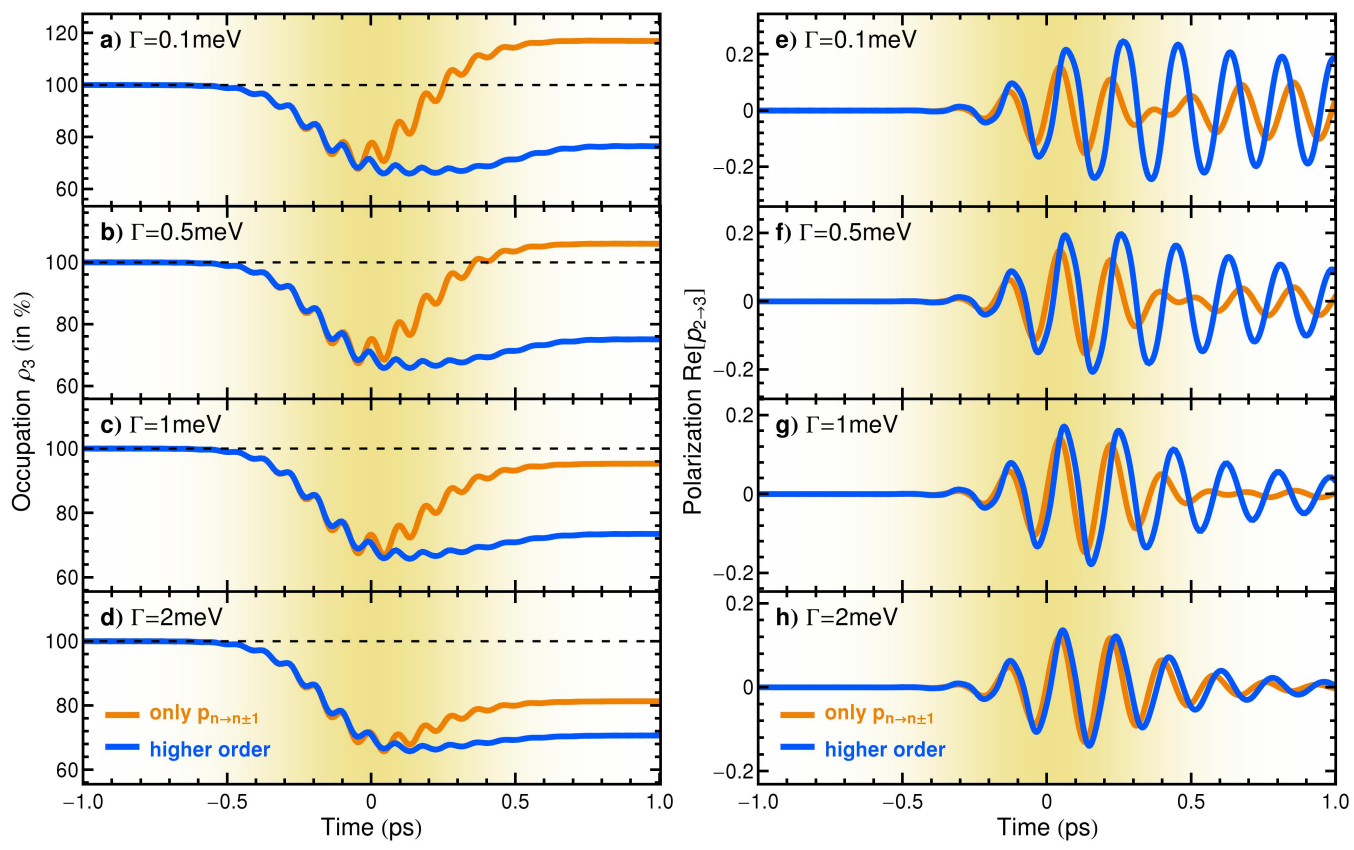

Figure 2. Temporal evolution of the carrier occupation $\rho_{3}(\mathrm{a})-(\mathrm{d})$ and the real part of the polarization $p_{2 \rightarrow 3}^{c c}(\mathrm{e})-(\mathrm{h})$ with and without the impact of higher-order coherences of the form $p_{n \rightarrow n \pm 2}$ and $p_{n \rightarrow n}^{v c}$ during an optical excitation with a width of $0.5 \mathrm{ps}$ (yellow shaded region). The situation corresponds to the case sketched in Fig. 1 with a resonant excitation of the inter-LL transition $2 \rightarrow 3$. Furthermore, the Fermi energy lies between $\mathrm{LL}_{3}$ and $\mathrm{LL}_{4}$ and the applied magnetic field corresponds to $4 \mathrm{~T}$. The orange lines show the carrier dynamics taking only into account the directly optically driven polarizations of the form $p_{n \rightarrow n \pm 1}$. In direct comparison, the blue lines show the carrier dynamics considering also higher-order polarizations. Results are shown for different values of the Landau level broadening $\Gamma$.

$$
\begin{aligned}
\dot{p}_{n \rightarrow n+2}^{v c}= & \left(i \triangle \omega_{n \rightarrow n+2}^{v c}-\gamma\right) p_{n \rightarrow n+2}^{v c} \\
+ & {\left[-\left(\Omega_{n \rightarrow n+1}^{v c}\right)^{*} p_{n+1 \rightarrow n+2}^{c c}+\left(\Omega_{n+1 \rightarrow n+2}^{v c} p_{n+1 \rightarrow n}^{v v}\right)^{*}+\left(\Omega_{n+1 \rightarrow n+2}^{c c}\right)^{*} p_{n \rightarrow n+1}^{v c}+\Omega_{n+1 \rightarrow n}^{v v} p_{n+1 \rightarrow n+2}^{v c}\right], } \\
\dot{p}_{n \rightarrow n-2}^{v c}= & \left(i \triangle \omega_{n \rightarrow n-2}^{v c}-\gamma\right) p_{n \rightarrow n-2}^{v c} \\
+ & {\left[-\left(\Omega_{n \rightarrow n-1}^{v c} p_{n-2 \rightarrow n-1}^{c c}\right)^{*}+\left(\Omega_{n-1 \rightarrow n-2}^{v c}\right)^{*} p_{n \rightarrow n-1}^{v v}-\Omega_{n-2 \rightarrow n-1}^{c c} p_{n \rightarrow n-1}^{v c}-\left(\Omega_{n \rightarrow n-1}^{v v}\right)^{*} p_{n-1 \rightarrow n-2}^{v c}\right] . }
\end{aligned}
$$

Here, we have negleted even higher-order polarizations of the form $p_{n \rightarrow n \pm 3}$.

\section{CARRIER DYNAMICS}

Now, we have everything at hand to investigate the impact of higher-order coherences on the carrier dynamics in Landau-quantized graphene. Solving the Bloch equations with and without the impact of the higher-order contributions $p_{n \rightarrow n}^{v c}$ and $p_{n \rightarrow n \pm 2}$, we can demonstrate their importance for the inter-Landau level dynamics.

We assume n-doped graphene with a Fermi energy of $\epsilon_{\mathrm{F}}=140 \mathrm{meV}$ that lies between $\mathrm{LL}_{3}$ and $\mathrm{LL}_{4}$ according to Fig. 1. The applied external magnetic field has the strength of $B=4 \mathrm{~T}$ and the temperature is set to $T=10 \mathrm{~K}$. The initial carrier occupations correspond to the Fermi-Dirac distribution at this temperature, while the initial value for polarizations is set to zero. Figure 2 shows the temporal evolution of the carrier occupation $\rho_{3}$ as well as the real part of the polarizations $p_{2 \rightarrow 3}^{v c}$, while the system is excited by a linearly polarized pump pulse with an energy matching the inter-LL transition $2 \rightarrow 3$, a pump fluence of $\epsilon_{\mathrm{pf}}=0.05 \mu \mathrm{Jcm}^{-2}$, and a width of $0.5 \mathrm{ps}$. The carrier dynamics is evaluated for different Landau level broadenings ranging from $\Gamma=0.1 \mathrm{meV}$ to 
$\Gamma=2 \mathrm{meV}$. Although the transition $2 \rightarrow 3$ is completely blocked, since the initial occupation of $\mathrm{LL}_{3}$ is $100 \%$, the occupation significantly changes during the optical excitation, cf. Figs. 2(a)-(e). We observe a decrease right after switching on the excitation pulse. This is attributed to off-diagonal pumping of the transition $3 \rightarrow 4$, cf. Fig. 1. This depopulation of $\mathrm{LL}_{3}$ enables a resonant excitation of the transition $2 \rightarrow 3$ after some time and as a result $\rho_{3}$ increases again. After the turning point of the occupation $\rho_{3}$, the transitions $2 \rightarrow 3$ and $3 \rightarrow 4$ have the same strength resulting in constant $\rho_{3}$. Note that $\rho_{3}$ shows clear oscillations at the beginning of the dynamics. Interestingly, their frequency does not correspond to the energy of the resonant optically allowed transition $2 \rightarrow 3$, but to the transition $2 \rightarrow 4$. This illustrates that this oscillation results from the interplay of the optically driven polarizations $p_{2 \rightarrow 3}^{c c}$ and $p_{3 \rightarrow 4}^{c c}$ inducing the higher-order coherence $p_{2 \rightarrow 4}^{c c}$.

The direct comparison of the carrier dynamics with and without taking into account higher-order polarizations reveals the rather large impact of these higher-order contributions, cf. blue and orange lines in Fig. 2(a)-(e). In fact, neglecting them results in a strongly unphysical behavior for small LL broadenings, where the occupation $\rho_{3}$ reaches values higher than 100\%, cf. Figs. 2(a),(b). For higher values of the LL broadening, the higher-order terms become less important. This is a consequence of the generally faster dephasing of the coherences, as illustrated in Figs. 2(e)-(h) showing the temporal evolution of the polarization $p_{2 \rightarrow 3}^{c c}$ with and without the impact of higher-order coherences. We find that the dephasing of the polarization significantly increases for enhanced Landau level broadenings. Furthermore, the influence of the higher-order coherences becomes smaller.

In summary, we have presented an investigation of the carrier-light interaction in Landau-quantized graphene based on optical Bloch equations. Our calculations reveal the crucial importance of higher-order coherences in specific situations, where the directly optically driven coherence is strongly suppressed due to Pauli blocking. We show that for short excitation pulses, very small Landau level broadenings, and certain configurations of magnetic field strength, Fermi energy, and excitation energy, higher-order coherences need to be taken into account to avoid unphysical behavior in the inter-Landau level carrier dynamics.

\section{ACKNOWLEDGMENTS}

We acknowledge financial support from the EU Graphene Flagship (CNECT-ICT-604391), the Swedish Research Council (VR), and the Deutsche Forschungsgemeinschaft (DFG) through SPP 1459. Furthermore, we thank A. Knorr (TU Berlin) and S. Winnerl (Helmholtz-Zentrum Dresden-Rossendorf) for inspiring discussions on the carrier dynamics in Landau-quantized graphene.

\section{REFERENCES}

1. Novoselov, K. S., Geim, A. K., Morozov, S. V., Jiang, D., Zhang, Y., Dubonos, S. V., Grigorieva, I. V., and Firsov, A. A., "Electric Field Effect in Atomically Thin Carbon Films," Science 306, 666-669 (2004).

2. Geim, A. K. and Novoselov, K. S., "The rise of graphene," Nat. Mater. 6(3), 183-191 (2007).

3. Geim, A. K., "Graphene: Status and Prospects," Science 324, 1530-1534 (2009).

4. Xia, F., Mueller, T., Lin, Y.-M., Valdes-Garcia, A., and Avouris, P., "Ultrafast graphene photodetector," Nature Nano. 4(12), 839-843 (2009).

5. Lin, Y.-M., Dimitrakopoulos, C., Jenkins, K. A., Farmer, D. B., Chiu, H.-Y., Grill, A., and Avouris, P., "100-GHz Transistors from Wafer-Scale Epitaxial Graphene," Science 327(5966), 662 (2010).

6. Bonaccorso, F., Sun, Z., Hasan, T., and Ferrari, A. C., "Graphene photonics and optoelectronics," Nature Photon. 4, 611-622 (2010).

7. Sun, Z., Hasan, T., Torrisi, F., Popa, D., Privitera, G., Wang, F., Bonaccorso, F., Basko, D. M., and Ferrari, A. C., "Graphene Mode-Locked Ultrafast Laser," ACS Nano 4(2), 803-810 (2010).

8. Avouris, P. and Dimitrakopoulos, C., "Graphene: synthesis and applications," Mater. Today 15(3), 86 - 97 (2012).

9. Gan, X., Shiue, R.-J., Gao, Y., Meric, I., Heinz, T. F., Shepard, K., Hone, J., Assefa, S., and Englund, D., "Chip-integrated ultrafast graphene photodetector with high responsivity," Nature Photon. 7(11), 883-887 (2013). 
10. Hu, S., Lozada-Hidalgo, M., Wang, F. C., Mishchenko, A., Schedin, F., Nair, R. R., Hill, E. W., Boukhvalov, D. W., Katsnelson, M. I., Dryfe, R. A. W., Grigorieva, I. V., Wu, H. A., and Geim, A. K., "Proton transport through one-atom-thick crystals," Nature 516, 227-230 (Dec. 2014).

11. Malic, E. and Knorr, A., [Graphene and Carbon Nanotubes: Ultrafast Optics and Relaxation Dynamics], Wiley-VCH (2013).

12. Kampfrath, T., Perfetti, L., Schapper, F., Frischkorn, C., and Wolf, M., "Strongly Coupled Optical Phonons in the Ultrafast Dynamics of the Electronic Energy and Current Relaxation in Graphite," Phys. Rev. Lett. 95, 187403 (2005).

13. Rana, F., "Electron-hole generation and recombination rates for Coulomb scattering in graphene," Phys. Rev. B 76, 155431 (2007).

14. Dawlaty, J. M., Shivaraman, S., Chandrashekhar, M., Rana, F., and Spencer, M. G., "Measurement of ultrafast carrier dynamics in epitaxial graphene," Appl. Phys. Lett. 92, 042116 (2008).

15. Sun, D., Wu, Z.-K., Divin, C., Li, X., Berger, C., de Heer, W. A., First, P. N., and Norris, T. B., "Ultrafast Relaxation of Excited Dirac Fermions in Epitaxial Graphene Using Optical Differential Transmission Spectroscopy," Phys. Rev. Lett. 101, 157402 (2008).

16. Rana, F., George, P. A., Strait, J. H., Dawlaty, J., Shivaraman, S., Chandrashekhar, M., and Spencer, M. G., "Carrier recombination and generation rates for intravalley and intervalley phonon scattering in graphene," Phys. Rev. B 79, 115447 (2009).

17. Winzer, T., Knorr, A., and Malic, E., "Carrier Multiplication in Graphene," Nano Lett. 10, 4839-4843 (2010).

18. Winnerl, S., Orlita, M., Plochocka, P., Kossacki, P., Potemski, M., Winzer, T., Malic, E., Knorr, A., Sprinkle, M., Berger, C., de Heer, W. A., Schneider, H., and Helm, M., "Carrier Relaxation in Epitaxial Graphene Photoexcited Near the Dirac Point," Phys. Rev. Lett. 107, 237401 (2011).

19. Breusing, M., Kuehn, S., Winzer, T., Malić, E., Milde, F., Severin, N., Rabe, J. P., Ropers, C., Knorr, A., and Elsaesser, T., "Ultrafast nonequilibrium carrier dynamics in a single graphene layer," Phys. Rev. B 83, 153410 (2011).

20. Kim, R., Perebeinos, V., and Avouris, P., "Relaxation of optically excited carriers in graphene," Phys. Rev. $B$ 84, 075449 (2011).

21. Winzer, T. and Malic, E., "Impact of Auger processes on carrier dynamics in graphene," Phys. Rev. B 85, 241404 (2012).

22. Winzer, T., Knorr, A., Mittendorff, M., Winnerl, S., Lien, M.-B., Sun, D., Norris, T. B., Helm, M., and Malic, E., "Absorption saturation in optically excited graphene," Appl. Phys. Lett. 101(22), 221115 (2012).

23. Malic, E., Winzer, T., and Knorr, A., "Efficient orientational carrier relaxation in optically excited graphene," Appl. Phys. Lett. 101(21), 213110 (2012).

24. Sun, D., Divin, C., Mihnev, M., Winzer, T., Malic, E., Knorr, A., Sipe, J. E., Berger, C., de Heer, W. A., First, P. N., and Norris, T. B., "Current relaxation due to hot carrier scattering in graphene," New J. Phys. 14(10), 105012 (2012).

25. Brida, D., Tomadin, A., Manzoni, C., Kim, Y. J., Lombardo, A., Milana, S., Nair, R. R., Novoselov, K. S., Ferrari, A. C., Cerullo, G., and Polini, M., "Ultrafast collinear scattering and carrier multiplication in graphene," Nature Commun. 4, 1987 (2013).

26. Gierz, I., Petersen, J. C., Mitrano, M., Cacho, C., Turcu, I. C. E., Springate, E., Stöhr, A., Köhler, A., Starke, U., and Cavalleri, A., "Snapshots of non-equilibrium Dirac carrier distributions in graphene," Nature Mater. 12(12), 1119-1124 (2013).

27. Winnerl, S., Göttfert, F., Mittendorff, M., Schneider, H., Helm, M., Winzer, T., Malic, E., Knorr, A., Orlita, M., Potemski, M., Sprinkle, M., Berger, C., and de Heer, W. A., "Time-resolved spectroscopy on epitaxial graphene in the infrared spectral range: relaxation dynamics and saturation behavior," J. Phys. Condens. Matter 25(5), 054202 (2013).

28. Johannsen, J. C., Ulstrup, S., Cilento, F., Crepaldi, A., Zacchigna, M., Cacho, C., Turcu, I. C. E., Springate, E., Fromm, F., Raidel, C., Seyller, T., Parmigiani, F., Grioni, M., and Hofmann, P., "Direct View of Hot Carrier Dynamics in Graphene," Phys. Rev. Lett. 111, 027403 (2013). 
29. Winzer, T., Malić, E., and Knorr, A., "Microscopic mechanism for transient population inversion and optical gain in graphene," Phys. Rev. B 87, 165413 (2013).

30. Kadi, F., Winzer, T., Malic, E., Knorr, A., Göttfert, F., Mittendorff, M., Winnerl, S., and Helm, M., "Microscopic Description of Intraband Absorption in Graphene: The Occurrence of Transient Negative Differential Transmission," Phys. Rev. Lett. 113, 035502 (2014).

31. Mittendorff, M., Winzer, T., Malic, E., Knorr, A., Berger, C., de Heer, W. A., Schneider, H., Helm, M., and Winnerl, S., "Anisotropy of Excitation and Relaxation of Photogenerated Charge Carriers in Graphene," Nano Lett. 14(3), 1504-1507 (2014).

32. Jago, R., Winzer, T., Knorr, A., and Malic, E., "Graphene as Gain Medium for Broadband Lasers," arxiv:1409.8182 (2014).

33. Plötzing, T., Winzer, T., Malic, E., Neumaier, D., Knorr, A., and Kurz, H., "Experimental Verification of Carrier Multiplication in Graphene," Nano Lett. 14(9), 5371-5375 (2014). PMID: 25144320.

34. Johannsen, J. C., Ulstrup, S., Crepaldi, A., Cilento, F., Zacchigna, M., Miwa, J. A., Cacho, C., Chapman, R. T., Springate, E., Fromm, F., Raidel, C., Seyller, T., King, P. D. C., Parmigiani, F., Grioni, M., and Hofmann, P., "Tunable Carrier Multiplication and Cooling in Graphene," Nano Lett. 15(1), 326-331 (2015). PMID: 25458168.

35. Winzer, T., Ciesielski, R., Handloser, M., Comin, A., Hartschuh, A., and Malic, E., "Microscopic View on the Ultrafast Photoluminescence from Photoexcited Graphene," Nano Lett. 15(2), 1141-1145 (2015).

36. Gierz, I., Calegari, F., Aeschlimann, S., Cervantes, M. C., Cacho, C., Chapman, R. T., Springate, E., Link, S., Starke, U., Ast, C. R., and Cavalleri, A., "Tracking primary thermalization events in graphene with photoemission at extreme timescales," arXiv:1506.00120 (2015).

37. Plochocka, P., Kossacki, P., Golnik, A., Kazimierczuk, T., Berger, C., de Heer, W. A., and Potemski, M., "Slowing hot-carrier relaxation in graphene using a magnetic field," Phys. Rev. B 80, 245415 (2009).

38. Mittendorff, M., Wendler, F., Malic, E., Knorr, A., Orlita, M., Potemski, M., Berger, C., de Heer, W. A., Schneider, H., Helm, M., and Winnerl, S., "Carrier dynamics in Landau-quantized graphene featuring strong Auger scattering," Nature Phys. 11, 75-81 (2015).

39. Wendler, F., Knorr, A., and Malic, E., "Resonant carrier-phonon scattering in graphene under Landau quantization," Appl. Phys. Lett. 103(25), 253117 (2013).

40. Wendler, F., Knorr, A., and Malic, E., "Carrier multiplication in graphene under Landau quantization," Nature Commun. 5, 3703 (2014).

41. Wendler, F. and Malic, E., "Population inversion in Landau-quantized graphene," arXiv:1410.2080 (2014).

42. Mittendorff, M., Orlita, M., Potemski, M., Berger, C., de Heer, W. A., Schneider, H., Helm, M., and Winnerl, S., "Intraband carrier dynamics in Landau-quantized multilayer epitaxial graphene," New J. Phys. 16, 123021 (2014).

43. Wang, Z.-W., Liu, L., and Li, Z.-Q., "Fast two-phonon relaxation process between the Landau levels of graphene on different polar substrates," Europhys. Lett. 108, 36005 (2014).

44. Goerbig, M. O., "Electronic properties of graphene in a strong magnetic field," Rev. Mod. Phys. 83, 11931243 (2011).

45. Lindberg, M. and Koch, S. W., "Effective Bloch equations for semiconductors," Phys. Rev. B 38, 3342-3350 (Aug 1988).

46. Haug, H. and Koch, S. W., [Quantum Theory of the Optical and Electronic Properties of Semiconductors], World Scientific (2009).

47. Kira, M. and Koch, S., "Many-body correlations and excitonic effects in semiconductor spectroscopy," Prog. Quantum Electron. 30(5), 155 - 296 (2006).

48. Rossi, F. and Kuhn, T., "Theory of ultrafast phenomena in photoexcited semiconductors," Rev. Mod. Phys. 74, 895-950 (Aug 2002).

49. Wendler, F., Funk, H., Mittendorff, M., Winnerl, S., Helm, M., Knorr, A., and Malic, E., "Efficient Auger scattering in Landau-quantized graphene," Proc. SPIE 9361, 936105-936105-7 (2015).

50. Rao, K. M. and Sipe, J. E., "Coherent photocurrent control in graphene in a magnetic field," Phys. Rev. $B$ 86, 115427 (2012). 
51. Castro Neto, A. H., Guinea, F., Peres, N. M. R., Novoselov, K. S., and Geim, A. K., "The electronic properties of graphene," Rev. Mod. Phys. 81, 109-162 (2009).

52. Jones, R. C., "A New Calculus for the Treatment of Optical Systems," J. Opt. Soc. Am. 31, 488-493 (1941).

53. Malic, E., Winzer, T., Bobkin, E., and Knorr, A., "Microscopic theory of absorption and ultrafast manyparticle kinetics in graphene," Phys. Rev. B 84, 205406 (2011). 